\title{
Entropic measure of wave-packet spreading and ionization in laser-driven atoms
}

\author{
J. B. Watson, ${ }^{1}$ C. H. Keitel, ${ }^{2}$ P. L. Knight, ${ }^{2}$ and K. Burnett ${ }^{1}$ \\ ${ }^{1}$ Clarendon Laboratory, Department of Physics, University of Oxford, Parks Road, Oxford OX1 3PU, United Kingdom \\ ${ }^{2}$ Blackett Laboratory, Optics Section, Imperial College, Prince Consort Road, London SW7 2BZ, United Kingdom
}

(Received 15 February 1996)

\begin{abstract}
We consider wave-packet spreading and ionization of a one-dimensional model hydrogen atom interacting with a strong high-frequency laser field in the stabilization domain. We demonstrate the effect of the pulse shape on the dynamics in phase space and visualize our results with both the Wigner and the $Q$ function phase-space quasiprobability representation. The Wehrl entropy is introduced and utilized as a convenient one-parameter measure of wave-packet spreading, phase-space localization, and ionization. [S10502947(96)02007-0]

PACS number(s): 32.80.Rm, 42.65.Ky, 42.50.Dv
\end{abstract}

\section{INTRODUCTION}

Atoms interacting with intense laser fields tend to fall apart through ionization, but although this ionization initially increases in rate with increasing intensity, provided frequencies and intensities are carefully chosen, the atom stabilizes. That is, the ionization rate decreases, rather than increases, with increasing intensity. Stabilization [1] can be observed by using high laser frequencies and high intensities. This phenomenon has been demonstrated for smooth pulse fields via both quantum-mechanical $[2,3]$ and classical [4] theoretical approaches and has recently been observed experimentally [5]. Despite the success of the classical models of this phenomenon, phase-space calculations have shown that stabilization may have intrinsically quantum-mechanical features $[6,7]$.

In a recent article [7] we identified quantum signatures in the stabilization dynamics by considering the Wigner function in phase space. The Wigner function [8] is well suited to this purpose since, although it superficially resembles a joint position-momentum phase-space distribution function, it may become negative, a clear indication of the quantum nature of the state under consideration. Wave packets are considered to be quasiclassical if they approach minimum uncertainty Gaussian wave packets, a sufficient condition if the Wigner function is to be positive over all of phase space [9]. In this paper we will consider two sources of negativity in the Wigner function. If we use an adiabatic turn-on, we will generate a single dressed eigenstate that will be much larger than a quasiclassical state and therefore nonclassical in nature. If, on the other hand, we use a nonadiabatic turn-on, then we will excite a coherent superposition of dressed eigenstates. The quantum interference between these dressed states is clearly nonclassical and therefore results in negative areas in the Wigner function. If the atom is subsequently ionized then the various parts of the wave function "fly apart" and this interference becomes small because the overlap of the wave packets contributing to this interference is reduced. For highly nonadiabatic pulses many dressed eigenstates are excited significantly: then the various interference contributions have a tendency to average out.

While the Wigner function is useful in investigating such quantum signatures, the negativities make it impossible to interpret the Wigner function as a probability distribution.
We therefore also consider a second phase-space distribution function, the Husimi or $Q$ representation [10], which is always positive. The Husimi distribution can be used to calculate an information-theoretic measure of the number of participating quasiclassical Gaussian wave packets. This measure is the Wehrl entropy [11] and, apart from interference terms, is determined by the number of Gaussian coherent states to "tile" its phase-space contour. The Wehrl entropy has been investigated and utilized by a group of authors [12]. It has recently been studied within a wider class of entropies that are based on a comparison of the wave function with an arbitrary basis of states in phase space [13]. This Wehrl entropy is directly related to the uncertainty area of the $Q$ function in phase space and it is appropriate as a measure of wave packet spreading and ionization. We will use the Wehrl entropy to study the effect of these phenomena in the stabilization domain using both an adiabatic $\sin ^{2}$ and a nonadiabatic trapezoidal pulse.

In the next section we will describe our numerical model, based on the solution of the time-dependent Schrödinger equation in one dimension. We will then investigate the stabilization dynamics for two different pulse shapes (one adiabatic, the other with a fast turn-on) using the Wigner function, before turning our attention to the $Q$ function and finally to the Wehrl entropy.

\section{NUMERICAL METHOD}

In this paper we present results based on the numerical solution of the one-dimensional time-dependent Schrödinger equation

$$
i \hbar \frac{\partial \psi(q, t)}{\partial t}=\left[-\frac{\hbar^{2}}{2 m} \frac{\partial^{2}}{\partial q^{2}}+V(q)+x E(t)\right] \psi(q, t) .
$$

Here $E(t)=E_{0} h(t) \cos (\omega t)$ is an electric field of peak amplitude $E_{0}$ modulated by an envelope function $h(t)$ and $V(q)$ is the potential due to the nucleus. We use the soft-core (or Rochester) potential [2] scaled so that its lowest eigenstate matches the binding energy of atomic hydrogen,

$$
V(q)=\frac{-e^{2}}{\sqrt{2 a_{0}^{2}+q^{2}}} .
$$


We use a one-dimensional atomic model since the calculation of three-dimensional phase-space distributions is not feasible with the current computational methods. The effect of using one-dimensional soft-core potentials rather than a three-dimensional Coulomb potential has been discussed elsewhere $[14,15]$.

In order to simplify the numerical calculation we transform into the Kramers-Henneberger (KH) frame, which moves with the ponderomotively driven electron, by making a unitary transform

$$
\begin{aligned}
\psi_{\mathrm{KH}}(q, t)= & U \psi(q, t) \\
= & \exp \left[\frac{i e}{m \hbar} \int_{0}^{T} d t^{\prime}\left[-A\left(t^{\prime}\right) p+e A^{2}\left(t^{\prime}\right) / 2\right]\right] \\
& \times \exp \left[\frac{i e}{\hbar} q A(t)\right] \psi_{\mathrm{KH}}(q, t) .
\end{aligned}
$$

This transform leads to a Schrödinger equation for the $\mathrm{KH}$ wave function of the form

$$
\hbar \frac{\partial \psi_{\mathrm{KH}}(q, t)}{\partial t}=\left[-\frac{\hbar^{2}}{2 m} \frac{\partial^{2}}{\partial q^{2}}+V(q+\alpha(t))\right] \psi_{\mathrm{KH}}(q, t)
$$

where

$$
\alpha(t)=-\frac{e}{m} \int_{0}^{t} d t^{\prime} A\left(t^{\prime}\right)
$$

is the displacement of a classical electron in the laser field. We integrate this equation numerically using the CrankNicholson method [2,3] in order to obtain the KH wave function at subsequent times: the length gauge wave function can then be obtained by applying the inverse unitary $\mathrm{KH}$ transform to the $\mathrm{KH}$ wave function.

The knowledge of the wave function as a function of time allows us to study the dynamics in phase space by calculating the Wigner function [8]

$$
\begin{aligned}
W(q, p, t)= & \frac{1}{\pi \hbar} \int_{-\infty}^{+\infty} d q^{\prime} \psi\left(q-q^{\prime}, t\right) \\
& \times \psi^{*}\left(q+q^{\prime}, t\right) \exp \left[2 i q^{\prime} p / \hbar\right] .
\end{aligned}
$$

One of the properties of this function is that it is Galilei invariant, i.e., displacing the wave function, either spatially or in momentum, simply shifts the Wigner function. Since the $\mathrm{KH}$ transform is just a combination of Galilean transforms, we can obtain the Wigner function in the length gauge by calculating the $\mathrm{KH}$ frame Wigner function and relabeling the axes [16].

By using phase-space representations rather than studying the wave function itself we can obtain information about the distribution both in space and also in momentum. Furthermore, as discussed above, the Wigner function may be negative in some regions of phase space, a signature of quantum behavior.
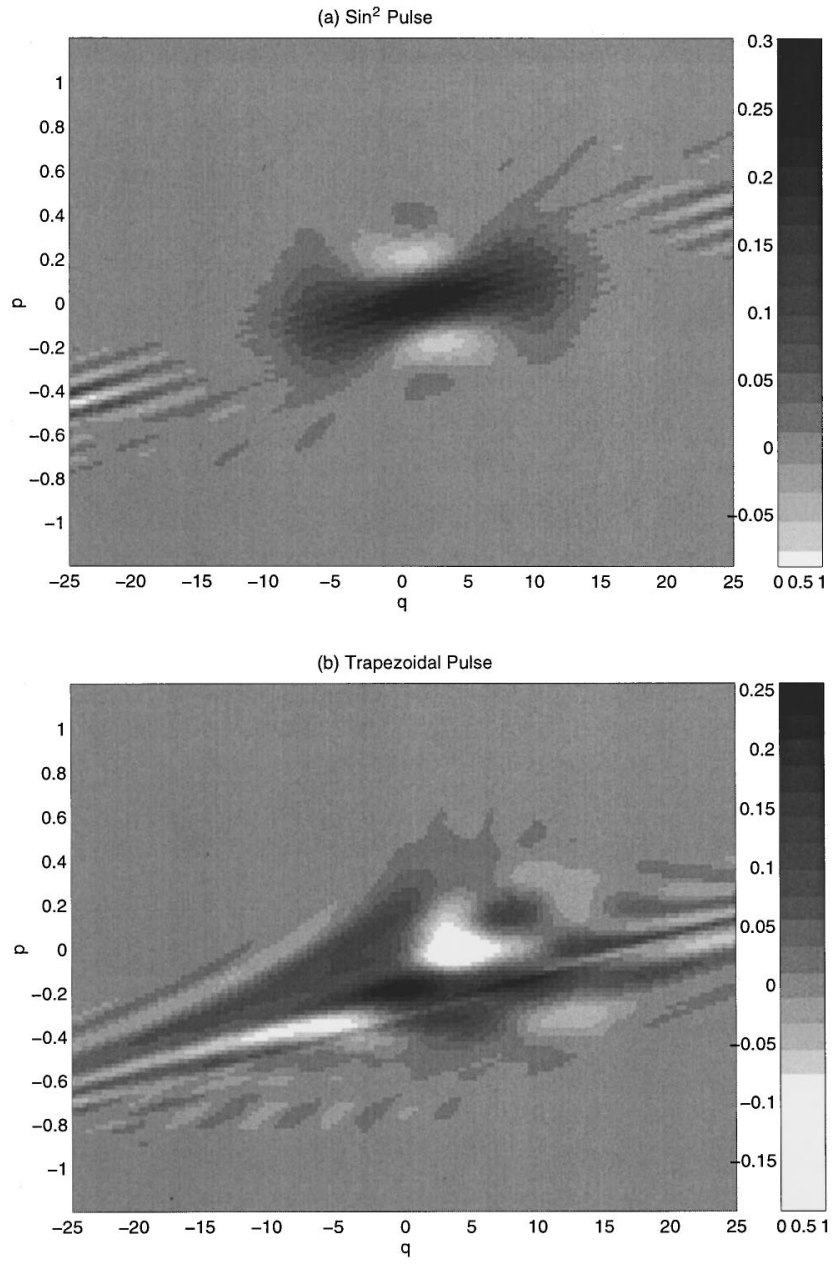

FIG. 1. Two-dimensional (2D) contour plot of the Wigner function calculated after 12 cycles of a 24-cycle pulse of frequency 1 a.u. and peak intensity 10 a.u. with (a) a $\sin ^{2}$ pulse shape and (b) a trapezoidal pulse shape with a two-cycle linear turn-on.

\section{PULSE SHAPE}

In our previous paper [7] we studied the phase-space dynamics of hydrogen undergoing stabilization in a nearly adiabatic $\sin ^{2}$ pulse. In this section we contrast this behavior with the phase-space dynamics for a trapezoidal pulse with a two-cycle linear turn-on. In other words we use the envelope functions

$$
h_{s}(t):= \begin{cases}\sin ^{2}[\omega t /(4 c)] & \text { if } 0<t<2 t_{\max }:=4 \pi c / \omega \\ 0 & \text { otherwise }\end{cases}
$$

where $\tau_{\max }$ is the length of the pulse and $c$ is the total number of cycles, and

$$
h_{t}(t):= \begin{cases}t / 2 \tau_{L} & \text { if } 0<t<2 \tau_{L} \\ 1 & \text { if } 2 \tau_{L}<t<22 \tau_{L} \\ \left(24 \tau_{L}-t\right) / 2 \tau_{L} & \text { if } 22 \tau_{L}<t<24 \tau_{L} \\ 0 & \text { otherwise }\end{cases}
$$

where $\tau_{L}$ is the laser period.

Figure 1 shows the Wigner functions calculated at the 
midpoint (also the peak intensity) of the smooth $\sin ^{2}$ [Fig. 1(a)] and trapezoidal Fig. 1(b)] pulses. In the trapezoidal case there is a very strong asymmetry in the phase-space distribution. This is a consequence of using a cosine field, with a very fast turn-on. If we consider the trajectory of a classical electron under the influence of such a field, starting from $q=0, p=0$ and at time $t=0$, its motion in the field will always be in the positive- $q$ region; the electron will be accelerated for a quarter of a period, come to rest after a quarter period, and then return to the nucleus, reaching $q=0$ with zero momentum after one full laser period has elapsed. This is equivalent, in the $\mathrm{KH}$ frame, to the core oscillating between $q=0$ and the minimum value of $q\left(-2 \alpha_{0}\right)$. Since in the $\mathrm{KH}$ reference frame the initial distribution (a peak at $q=0$ ) is strongly asymmetric about the center of the core oscillation, the wave function and therefore the phase-space distributions are asymmetric throughout the pulse evolution.

In the $\sin ^{2}$ case, the Wigner function contains two strongly negative lobes arranged on either side of the main peak. In a previous paper [7] we demonstrated that this distribution is due to the formation of a superposition of symmetric eigenstates of the time-averaged $\mathrm{KH}$ potential, a consequence of the smooth adiabatic turn-on. It is well established that such superpositions give rise to Wigner functions with symmetrical negativities [17]. In the trapezoidal case the distribution is very different, and rather than the two strongly negative areas, we only find a single negative area. Since the trapezoidal pulse has such a fast turn-on we excite a far greater population into the higher $\mathrm{KH}$ eigenstates. As a result of both the greater spatial structure of the higher $\mathrm{KH}$ eigenstates and the interferences between the various eigenstates, we find a far more complicated structure in the Wigner function.

In order to confirm the above interpretation of our Wigner functions, we have calculated the populations in the first seven KH eigenstates for both pulse shapes; the results are shown in Table I.

From these results it is apparent that for the $\sin ^{2}$ pulse, the wave function is dominated by the adiabatic $\mathrm{KH}$ ground state, exactly as one would expect for a smooth turn-on. There is also a small population in the second excited state, about $8 \%$, with less than $0.3 \%$ of the population in the remaining bound states. Similar populations are found throughout the pulse, although during the tail of the pulse there is a significant transfer of population into the second excited state: at the end of the pulse there is a population of 0.59 in the ground state compared with 0.24 in the second excited state. We note that there are considerably lower

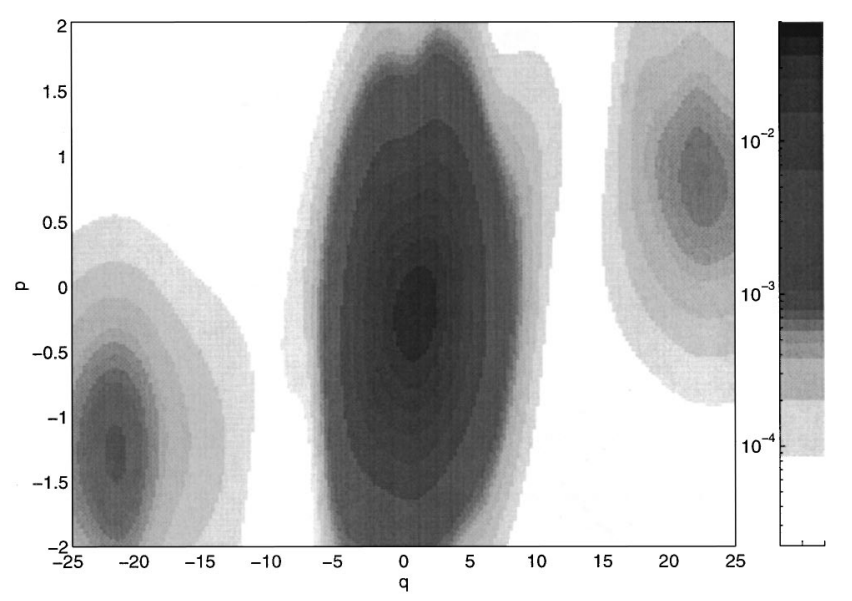

FIG. 2. 2D contour plot of the Husimi $Q$ function calculated after 6 cycles of a 24 -cycle $\sin ^{2}$ pulse of peak intensity 10 a.u. and frequency 1 a.u.

populations in the odd-parity states, which is the reason for the symmetry observed in Fig. 1(a). For the trapezoidal case the situation is very different. The population in the lowest $\mathrm{KH}$ eigenstate is less than $10 \%$, with higher populations in the next three states. In fact, there is significant population right up to the sixth excited state. The asymmetry in the Wigner function for this case is reflected by the equal populations in the odd- and even-parity states. These populations remain approximately constant throughout the pulse, as one would expect in the stabilization regime. Finally, we point out that for the $\sin ^{2}$ pulse the ionization is much smaller than for the trapezoidal case. We shall return to these differences in the bound state population when we consider the Wehrl entropy.

\section{IV. $Q$ FUNCTIONS}

In the preceding section we have seen that the Wigner function is a very convenient diagnostic for the identification of quantum behavior within a system. For the following discussion we turn to a closely related phase-space quasiprobability function that is always positive, called the $Q$ or $\mathrm{Hu}-$ simi function [10]. The $Q$ function has an appealing interpretation in quantum measurement in terms of "propensities" [18]: Let us represent our state $\psi$ by its Wigner function $W_{\psi}$. One may only obtain information about the system via comparison with a measurement device $\phi$, so that the measurement output may be described by a new phase-space

TABLE I. Calculated population in the first seven KH eigenstates for the $\sin ^{2}$ and trapezoidal pulses.

\begin{tabular}{ccc}
\hline \hline KH eigenstate & $\sin ^{2}$ pulse & Trapezoidal pulse \\
\hline ground & 0.8293 & 0.0947 \\
first excited & $1.122 \times 10^{-4}$ & 0.1983 \\
second excited & 0.0836 & 0.2191 \\
third excited & $4.9771 \times 10^{-5}$ & 0.2090 \\
fourth excited & 0.0028 & 0.0947 \\
fifth excited & $3.5041 \times 10^{-5}$ & 0.0041 \\
sixth excited & $1.2321 \times 10^{-4}$ & 0.0250 \\
bound-state population & 0.916 & 0.8449 \\
\hline \hline
\end{tabular}



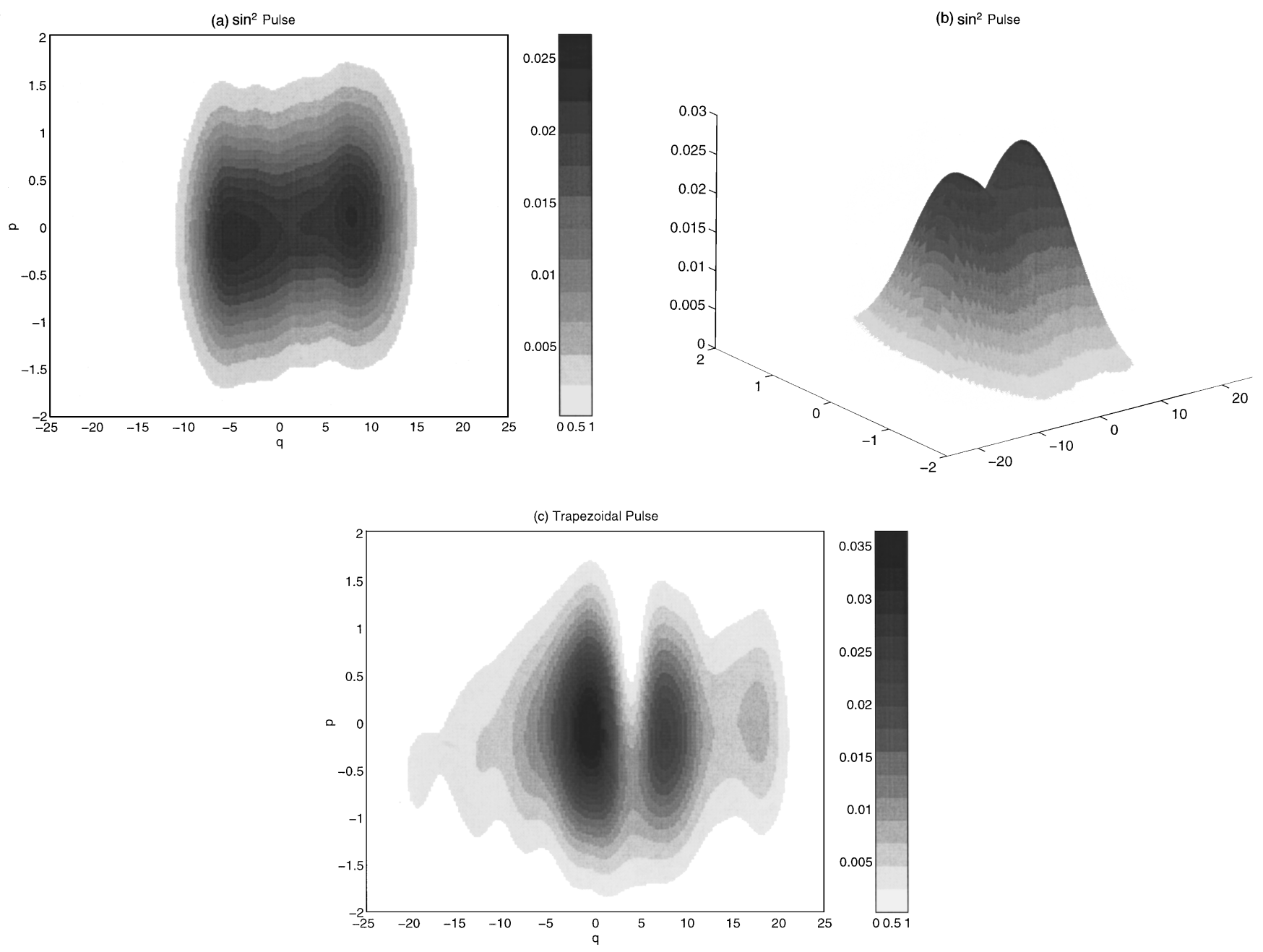

FIG. 3. (a) 2D contour plot, (b) 3D surface plot of the Husimi $Q$ function calculated after 12 cycles of a 24 -cycle sin ${ }^{2}$ pulse of peak intensity 10 a.u. and frequency 1 a.u, and (c) 2D contour plot of the $Q$ function calculated after 12 cycles of a trapezoidal pulse using the same parameters.

distribution via the convolution [18]

$$
P(q, p)=\int d q^{\prime} d p^{\prime} W_{\psi}\left(q^{\prime}, p^{\prime}\right) W_{\phi}\left(q^{\prime}-q, p^{\prime}-p\right) \text {. }
$$

If the measurement device can be represented by a coherent state, i.e., the Gaussian wave packet

$$
W_{\phi}\left(q^{\prime}, p^{\prime}\right)=\frac{1}{\pi \hbar} \exp \left[-q^{\prime 2}-\left(\frac{p^{\prime}}{\hbar}\right)^{2}\right],
$$

displaced in phase space, then the phase-space distribution relevant to the measurement $P(q, p)$ is the positive Husimi or $Q$ function. Although we have chosen a minimum uncertainty state as our reference wave packet, the "smearing", due to the measurement is already sufficient to remove any negativity from the distribution.

In Fig. 2 we show a two-dimensional representation of the $Q$ function after six cycles of the $\sin ^{2}$ pulse. In this case the distribution is dominated by a strong peak at the center. There is no dichotomy and very little spreading of the wave packet, a consequence of the fact that the maximum displacement $\alpha$ is still relatively small at this stage of the pulse.
In this plot we have chosen a logarithmic scale in order to emphasize the outgoing part of the distribution, at the expense of detail at the core. On this scale we can see peaks in the top right and bottom left areas of the plot, corresponding to ionization: i.e., in addition to the momentum due to the oscillation of the field, these wave packets also have a drift velocity and will therefore leave the nucleus.

We have calculated the $Q$ function for both the $\sin ^{2}$ and trapezoidal pulses after 12 cycles (at the peak of the $\sin ^{2}$ pulse). In Fig. 3 we have displayed the $Q$ function for the $\sin ^{2}$ pulse both as a two-dimensional contour plot [Fig. 3(a)] and also as a three-dimensional surface plot [Fig. 3(b)]; we also display a contour plot of the $Q$ function for the trapezoidal pulse [Fig. 3(c)]. In the adiabatic case, there is a very clear dichotomy in the $Q$ function. This dichotomy is, of course, directly related to the splitting of the wave function in the high-frequency stabilization regime, an effect that has previously been described by many authors in the wave function representation $[2,3]$. The presence of the dichotomy suggests that the wave function generated under these circumstances is dominated by the lowest $\mathrm{KH}$ eigenstates, although we should emphasize that the degree of dichotomy depends on the relative phase between the superposed $\mathrm{KH}$ eigen- 
states. In the trapezoidal case, we once again find a strong asymmetry, with three main peaks in the distribution. This extra structure is due to the superposition of a number of $\mathrm{KH}$ eigenstates. We note that the negativity clearly visible in the Wigner representations of Fig. 1 has not survived the smoothing generated in the creation of the $Q$ function from the Wigner function.

We have also calculated the $Q$ function at the end of the pulse. Here, as one would expect, there is a single sharp peak centered on the origin. Again, if we magnify the probability scale we see ionizing wave packets leaving the nucleus.

From these plots we deduce essentially two points. First, at the peak of the $\sin ^{2}$ pulse we generate a superposition of a small number of $\mathrm{KH}$ eigenstates, which, due to the relative phase between the two states at this particular stage in the pulse, results in a clear dichotomy. Second, at the start and end of the pulse the intensity is lower than that required for stabilization and therefore there is substantial ionization of the atom. This is consistent with our observations based on the Wigner function, in which we clearly see the outgoing ionization waves.

At this point we should emphasize that much of this information, in particular the nature of the ionizing wave packet, can also be directly visualized in the wave-function representation. However, the phase-space picture also gives us simultaneous information about the momentum distribution of the wave packet, so that we are able to visualize directly in which direction and how fast parts of the wave packet are moving.

\section{ENTROPY}

The $Q$ function can be used directly to provide a measure of the number of participating states in the dynamics. We can define the information-theoretic Wehrl entropy [13] by

$$
S_{W}(t)=-\frac{k_{B}}{\hbar} \int d p d q Q(q, p, t) \ln Q(q, p, t),
$$

where $Q(q, p)=\hbar[P(q, p)]$ is a dimensionless form of the $Q$ function. From now on the Boltzmann constant $k_{B}$ and $\hbar$ will be assumed to be equal to unity. The Wehrl entropy is not a measure of the purity of the wave function as is the von Neumann entropy nor should it be regarded as a thermodynamic entropy. Rather, it can be interpreted as the number of coherent states one requires to "cover up" the state of the system in phase space, or more precisely to cover the uncertainty area. In fact, in the case when the $Q$ function can be represented by a Gaussian distribution of contour area $A$, at a height $1 / e$ of the maximum, then the Wehrl entropy is given by [13]

$$
S_{W}=1+\ln [A] .
$$

If the atomic wave packet is a single Gaussian of minimum uncertainty width, then $S_{W}$ attains its minimum value of $1+\ln (2 \pi)$. If the wave packet consists of $N$ separate, nonoverlapping coherent-state components, then $S_{W}=1+\ln [2 \pi N]$, whereas if they are overlapping and interfering, then $S_{W}$ is reduced below this value.

In Fig. 4 we have plotted the Wehrl entropy as a function of the number of cycles of the laser pulse. The entropy increases over the first part of the pulse and reaches a maxi-

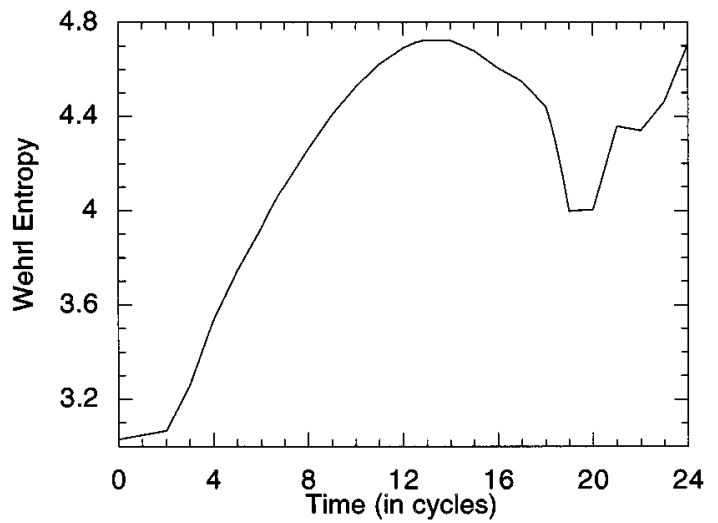

FIG. 4. Wehrl entropy as a function of time for a 1D model hydrogen atom in a 24 -cycle $\sin ^{2}$ laser pulse of peak field 10 a.u. and frequency 1 a.u.

mum just after the peak in the laser intensity. The entropy then decreases for approximately four cycles before increasing during the tail of the pulse. In order to explain this behavior we need to consider contributions to the entropy from two distinct sources: the "core" (the bound part) of the wave function, and the ionizing wave packets. Let us first consider the stabilized part of the wave function. As we have previously discussed, the bound part of the wave function is made up of a coherent superposition of a small number of $\mathrm{KH}$ eigenstates. We will therefore start by considering the entropy of a $\mathrm{KH}$ eigenstate as a function of the maximum displacement $\alpha_{0}$. In this high-frequency regime we assume that the dressed states are the eigenfunctions of the time averaged $\mathrm{KH}$ potential, given by

$$
V_{0}\left(\alpha_{0}, q\right)=\frac{1}{\pi} \int_{-1}^{+1} d u \frac{V\left(q+\alpha_{0} u\right)}{\sqrt{1-u^{2}}}
$$

The shape of this potential, and in particular the width, depends critically on the value of $\alpha$ [19]. For increasing values of $\alpha$ the potential becomes more shallow and has a greater spatial extent. As a consequence its eigenstates have a greater spatial spread and therefore a greater entropy (i.e., they are made up of more and more minimum-uncertainty

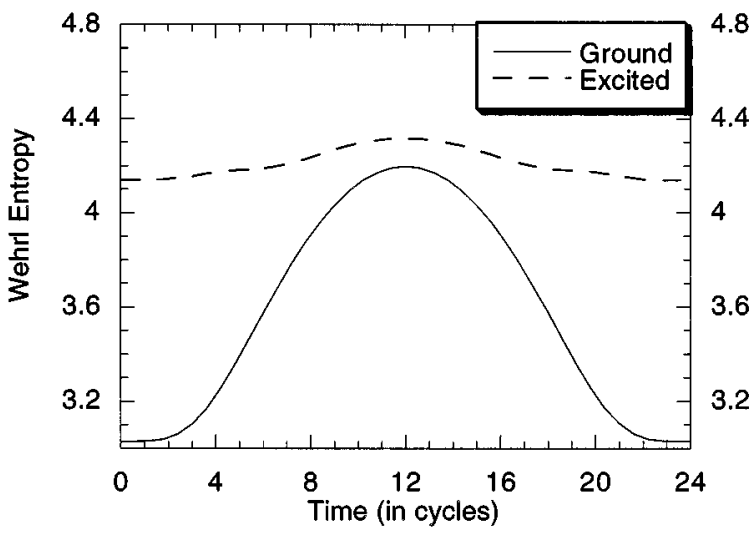

FIG. 5. Wehrl entropy of the two lowest even-parity eigenstates of the time-averaged $\mathrm{KH}$ potential as a function of time where $\alpha=\sin ^{2}(\omega t / 24 \pi)$. 
Gaussian component packets). In Fig. 5 we have plotted the entropy of the lowest $\mathrm{KH}$ eigenstate and also of the next even-parity state (from our previous analysis we know that these are the dominant states) for various values of $\alpha$. We have plotted this against the number of cycles of our pulse at which the field corresponds to that value of $\alpha$. For the ground (lowest-energy) state (solid curve) there is a very strong dependence on the value of $\alpha$. This is exactly as one would expect since we start at the beginning of the pulse $\alpha=0$ where the probability density has a single peak. As the field, and therefore $\alpha$, increases the wave function becomes broader and starts to show a dichotomy; this broadening is naturally accompanied by an increase in entropy. In the case of the excited state there is a much slower dependence, since even with no field the probability distribution is split into separated nodes, which we can (approximately) regard as discrete packets. We have already demonstrated that for the adiabatic pulse shape the ground state dominates the wavepacket evolution and therefore it is not surprising that the entropy in this case follows the pattern of the ground state. At this point we note that in reality we will have a coherent superposition of KH eigenstates; we will return to the effect of such superpositions later.

The second contribution to the Wehrl entropy is due to ionization of the atom. At the start of the pulse, the intensity is too low for stabilization to occur. As we are in a highfrequency stabilization domain where hydrogen can be ionized by a single photon and the field amplitude is still high, we see strong ionization on the rising edge of the pulse, before the stabilization regime is reached. The same argument is also true at the tail of the pulse. Our calculation of the bound-state populations indicates that for the parameters chosen here there is approximately $8 \%$ depletion during the first six cycles, $6 \%$ depletion over the final six cycles, but less than $1 \%$ during the central twelve cycles, where the pulse maximum is reached. We therefore get a sharp increase in entropy over the first few cycles due to ionization, i.e., an emergent flux of the probability emanating from the core. This is followed by a slower change in the Wehrl entropy due to the spreading of the freely evolving ionizing wave functions. Over the final cycles there is another sharp increase due to further ionization.

As we have already seen, if we use a trapezoidal laser pulse, the rapid turn-on leads to a much higher probability of excitation, both to the higher-energy KH eigenstates and also into the continuum (i.e., ionization). This depletion of the ground state during the turn-on is reflected in the entropy (Fig. 6). Once again it is convenient to consider contributions due to the bound and ionizing parts of the wave packet separately. In this case we turn the field on rapidly, so after the first couple of cycles the value of $\alpha$ is constant. There are, however, still changes in the entropy due to the fact that we have a superposition of states. Even though, at the peak of the pulse, the probabilities of each of these states are approximately constant, each state will have a phase that oscillates with its own characteristic frequency. As a result, the various eigenstates beat together, causing the wave packet to "breathe." Since this breathing causes changes to the spatial extent of the wave packet, it leads to fluctuations in the entropy (we will demonstrate this effect for a superposition of two eigenstates in Sec. V A). As with the $\sin ^{2}$ pulse there is

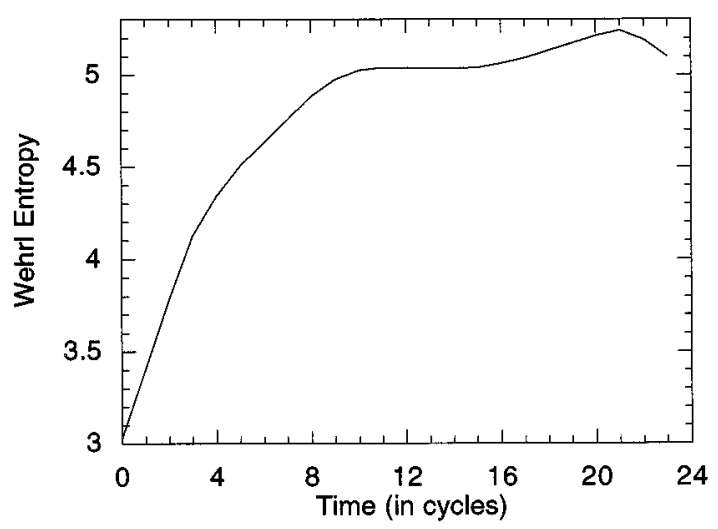

FIG. 6. Wehrl entropy as a function of time for a 1D model hydrogen atom in a 24-cycle trapezoidal laser pulse of peak field 10 a.u., frequency 1 a.u., and two-cycle linear turn-on.

also a contribution due to ionization. During the turn-on there is quite significant ionization, which leads to a rapid increase in the entropy over the first six cycles, followed by a slow increase due to the spreading of the outgoing wave packets.

We have therefore explained the entropy for both the $\sin ^{2}$ and trapezoidal pulse shapes in terms of ionization during the turn-on followed by dispersion of the ionizing wave packets, plus spreading of the bound part of the wave function. In the $\sin ^{2}$ case there is an increase in entropy due to the broadening of the wave function with increasing $\alpha$, while for the trapezoidal case there are fluctuations due to the different eigenstates beating together. Finally we point out that in the trapezoidal case there is a higher ionization probability and therefore a higher entropy. In this section we will consider the entropy of a coherent superposition of $\mathrm{KH}$ eigenstates in more detail.

\section{A. Entropy of superposition states}

In our previous analysis of the entropy we have assumed that, for the $\sin ^{2}$ laser pulse shape case at least, the behavior of the stabilized wave packet can be explained in terms of the Wehrl entropy of the ground state. However, in both this paper and our previous paper we have demonstrated that a coherent superposition of KH eigenstates is formed. In this section we will briefly consider the effect of such a superposition on the Wehrl entropy. In particular we will show that the entropy of the superposition depends on the relative phase of the states. In order to demonstrate this effect we have calculated the entropy of a superposition of the ground and second excited KH states with weights $a$ and $b$ and a relative phase $\theta$,

$$
|\psi\rangle=a\left|g_{k h}\right\rangle+b e^{i \theta}\left|2_{k h}\right\rangle .
$$

In Fig. 7 we have plotted the entropy of such a superposition, for $\alpha=10$ with relative weights identical to those calculated at the peak of our $\sin ^{2}$ pulse, i.e., $a^{2}=0.84$ and $b^{2}=0.08$, with values of $\theta$ in the range $0-2 \pi$. There is a definite dependence on phase, with a minimum entropy when $\theta=\pi$. This can be explained by considering the wave function formed by the superposition. The effect of varying the phase is to cause the wave function to breathe, increasing or 


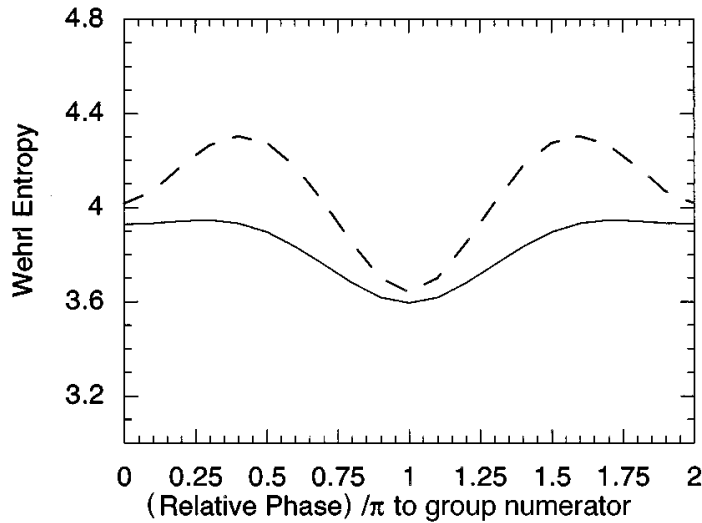

FIG. 7. Wehrl entropy of a superposition of the two lowest even-parity $\mathrm{KH}$ eigenstates as a function of their relative phase, using a weighting similar to that found in the adiabatic case (solid line) and also an equal weighting (dashed line).

decreasing the dichotomy (and also the spatial extent) of the probability distribution and therefore changing the entropy. A similar effect has been observed for a superposition of two harmonic oscillator states [13]. As one might expect, this effect is greater when both states are present with approximately equal weighting. We have plotted the entropy for this case as a dashed line in Fig. 7. A sudden turn-on will generate such $\mathrm{KH}$ superpositions that can destructively or constructively interfere, changing dramatically the size of phase space occupied by the wave packet.

\section{CONCLUSION}

In this paper we have used several distinct phase-space approaches to contrast stabilization using $\sin ^{2}$ and trapezoidal pulse shapes. We have shown that for an adiabatic pulse shape we dominantly populate the lowest $\mathrm{KH}$ eigenstate, with a small population in the next even parity excited state, whereas for a trapezoidal pulse shape with a rapid turn on we populate several $\mathrm{KH}$ eigenstates with approximately equal probability. Furthermore we have demonstrated that the number of populated states has a very clear effect on both the Wigner and Husimi phase-space distributions. Finally we have used the Wehrl entropy as a measure of the wavepacket spreading, and explained the results obtained in terms of ionization of the atom and the formation of $\mathrm{KH}$ eigenstates.

\section{ACKNOWLEDGMENTS}

The financial support of the European Community and of the United Kingdom Engineering and Physical Sciences Research Council is gratefully acknowledged.
[1] J. J. Gersten and M. H. Mittleman, J. Phys. B 9, 2561 (1976); M. Gavrila and J. Z. Kaminski, Phys. Rev. Lett. 65, 2362 (1984); M. Gavrila et al., in Atoms in Intense Laser Fields, edited by M. Gavrila (Academic, Boston, 1992); K. Burnett, V. C. Reed, and P. L. Knight, J. Phys. B 26, 561 (1993).

[2] J. H. Eberly, Q. Su, and J. Javanainen, Phys. Rev. Lett. 62, 881 (1989); Q. Su, J. H. Eberly, and J. Javanainen, ibid. 64, 862 (1990); Q. Su and J. H. Eberly, Phys. Rev. A 43, 2474 (1991); J. H. Eberly et al., in Super-Intense Laser-Atom Physics, Vol. 316 of NATO Advanced Study Institute, Series B: Physics, edited by B. R. M. Piraux, A. L'Huillier, and K. Rzazewski (Plenum, New York, 1993); Q. Su, A. Sanpera, and L. RosoFranco, Int. J. Mod. Phys. B 8, 1655 (1994).

[3] V. C. Reed, P. L. Knight, and K. Burnett, Phys. Rev. Lett. 67, 1415 (1991); V. C. Reed, K. Burnett, and P. L. Knight, Phys. Rev. A 47, R34 (1993); P. L. Knight et al., in Super-Intense Laser-Atom Physics (Ref. [2]).

[4] J. Grochmalicki, M. Lewenstein, and K. Rzazewski, Phys. Rev. Lett. 66, 1038 (1991); R. Grobe and C. K. Law, Phys. Rev. A 44, R4141 (1991); M. Gajda et al., ibid. 46, 1638 (1992); B. Sundaram and R. V. Jensen, ibid. 47, 1415 (1993); F. Benvenuto et al., ibid. 47, R786 (1993); C. H. Keitel, P. L. Knight, and K. Burnett, Europhys. Lett. 24, 539 (1993); C. H. Keitel and P. L. Knight, Phys. Rev. A 51, 1420 (1995).

[5] M. P. de Boer, J. H. Hoogenraad, R. B. Vrijen, L. D. Noordam, and H. G. Muller, Phys. Rev. Lett. 71, 3263 (1993).

[6] J. Bestle, V. A. Akulin, and W. P. Schleich, Phys. Rev. A 48, 746 (1993).
[7] J. B. Watson, C. H. Keitel, P. L. Knight, and K. Burnett, Phys. Rev. A 52, 4023 (1995).

[8] E. P. Wigner, Phys. Rev. 40, 749 (1932).

[9] R. L. Hudson, Rep. Math. Phys. 6, 249 (1974).

[10] K. Husimi, Proc. Phys. Math. Soc. Jpn. 22, 264 (1940); see also Y. Kano, J. Math. Phys. 6, 1913 (1965).

[11] A. Wehrl, Rev. Mod. Phys. 50, 221 (1978); Rep. Math. Phys. 16, 353 (1979).

[12] C. H. Keitel and K. Wódkiewicz, Phys. Lett. A 167, 151 (1992); A. Orlowski, Phys. Rev. A 48, 727 (1993); A. Anderson and J. J. Halliwell, Phys. Rev. D 48, 2753 (1993).

[13] V. Bužek, C. H. Keitel, and P. L. Knight, Phys. Rev. A 51, 2575 (1995); 51, 2594 (1995).

[14] T. Ménis, R. Taieb, V. Véniard, and A. Maquet, J. Phys. B 25, L263 (1992).

[15] T. Millack, J. Phys. B 26, 4777 (1993).

[16] We wish to point out that in our previous paper [7] the Wigner functions are plotted in the $\mathrm{KH}$ frame, despite the inference that they are in the length gauge. As we have pointed out in this paper, the only difference is a shift in the axes.

[17] V. Bužek and P. L. Knight, in Progress in Optics, edited by E. Wolf (North-Holland, Amsterdam, 1996), Vol. 34, p. 1.

[18] Y. Aharonov, D. Z. Albert, and C. K. Au, Phys. Rev. Lett. 47, 1029 (1982); R. F. O'Connell and A. K. Rajogopal, ibid. 48, 525 (1982); K. Wódkiewicz, ibid. 52, 1064 (1984); Phys. Lett. A 115, 304 (1986); 129, 1 (1988).

[19] Valerie Reed, D. Phil. Thesis, University of Oxford, 1991 (unpublished). 